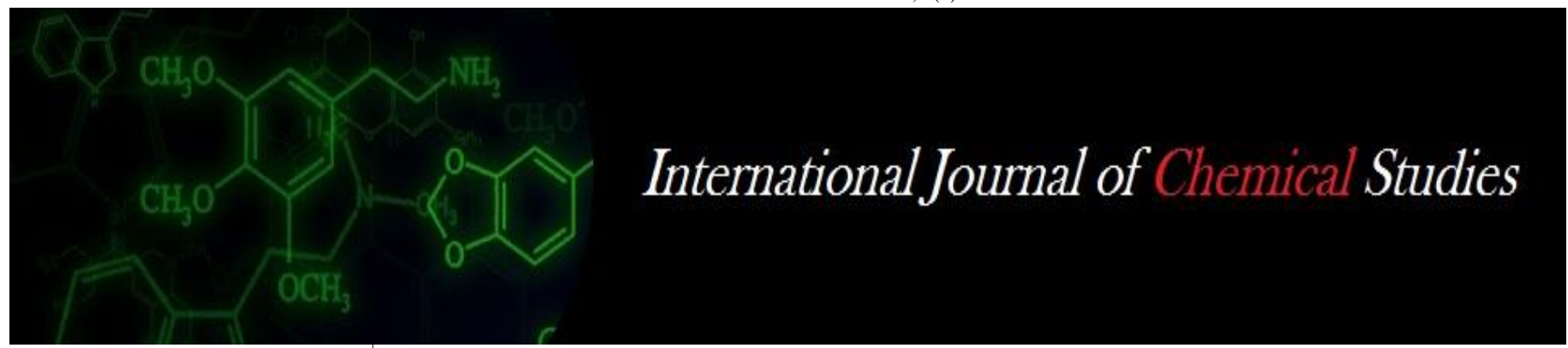

P-ISSN: 2349-8528

E-ISSN: 2321-4902

www.chemijournal.com

IJCS 2021; 9(1): 2057-2060

(C) 2021 IJCS

Received: 21-11-2020

Accepted: 30-12-2020

\section{Pramod Kumar}

Department of Agrometeorology,

College of Agriculture, IGKV,

Raipur, Chhattisgarh, India

\section{GK Das}

Department of Agrometeorology, College of Agriculture, IGKV,

Raipur, Chhattisgarh, India

\section{Deepak Kumar}

Department of Agrometeorology, College of Agriculture, IGKV,

Raipur, Chhattisgarh, India
Corresponding Author:

Pramod Kumar

Department of Agrometeorology,

College of Agriculture, IGKV,

Raipur, Chhattisgarh, India

\title{
Studies on heat unites on various phenophases of wheat (Triticum aestivum) under different growing environment
}

\section{Pramod Kumar, GK Das and Deepak Kumar}

DOI: $\underline{\text { https://doi.org/10.22271/chemi.2021.v9.i1ac.11526 }}$

\begin{abstract}
The present investigation entitled "Studies on Heat unites on various phenophases of wheat (Triticum aestivum) under different growing environment" was carried out during Rabi season of 2016-2017, at the Research Farm of Indira Gandhi Krishi Vishwavidhyalaya, Raipur to find out the wheat varieties under late sowing conditions in a rice based cropping system. The treatment combinations of three sowing dates ( $1^{\text {st }}$ December, $11^{\text {th }}$ December and $21^{\text {st }}$ December,) and three varieties (Ratan, GW-273, and GW-366) were laid out in a randomized block design with three replication. The value of GDD, PTU, HTU, RUE and HTU for different varieties were recorded and maximum value in these parameter was found highest in GW-273 under D1 ( $1^{\text {st }}$ December) growing environment.
\end{abstract}

Keywords: Heat unites (GDD, PTU, THU, HUE, RUE), varieties (Ratan, GW-273, GW-366), yield, dates of sowing

\section{Introduction}

Wheat is the world's number one cereal crop after rice, grown under diverse agro-climatic conditions, contributing nearly one-third of total food grains production. This grown is not only in the temperate zone but also in tropical and sub-tropical zone tropical and sub-tropical zones. It can be cultivated as high as 3500 meters above mean sea level. Major wheat producing countries of the world are China, India, United States of America, France, Russia, Canada, Germany, Turkey, Australia, Ukraine, and Pakistan. In the world, an area under cereals is 685.67 million hectare with the production of 2239.39 metric tons and productivity $3265.98 \mathrm{~kg}$ ha-1. In India crop is grown in an area of 302.27 lakh ha. with the production 93.50 million tons and productivity of $3093 \mathrm{~kg}$ ha-1. (Annual report 2015-16) ${ }^{[2]}$.

Worldwide, wheat provides nearly 55\% of the carbohydrates and $20 \%$ of the food calories consumed globally (Breiman and Graur, 1995) ${ }^{[4]}$. It is an annual plant that belongs to the grass family Poaceae, tribe Triticeae, and subtribe Triticineae. It is thought to have originated on the Eurasian continent, a starting point from which man spread it throughout the world, including China and Central Europe (Haider, 2012).

Three main species commonly grown in the world including India are the common wheat (Triticum aestivum), Marconi or durum wheat (T. durum) and emmer wheat (T. dicoccum) maximum area are covered by $T$. aestivum out of 3 species. In India, more than $80 \%$ of the total wheat area is under this species where as $12 \%$ and $1 \%$ area under Marconi and emmer wheat. (Draganka et al, 2004).

India has a large area under wheat and about $90 \%$ of total wheat production is contributed by five states viz., Uttar Pradesh, Punjab, Haryana, Madhya Pradesh, and Rajasthan. The other wheat producing states are Bihar, Gujarat, Jammu and Kashmir, Maharashtra, West Bengal and Chhattisgarh. India the total food grain production for the year 2004 was 212.05 million tons and wheat occupies an area of 2.63 million hectares with the production of 7.2 millions tons and productivity of $2716.98 \mathrm{~kg}$ ha-1 (Anonymous, 2010) ${ }^{[3]}$.

\section{Material and Methods}

The field experiment was carried out at the Research and Instructional farm of Indira Gandhi Krishi Vishwavidyalaya; 
Raipur situated in Eastern Central part of Chhattisgarh at latitudes of $21^{0} .16^{\prime} \mathrm{N}$, longitude $81^{\circ} .36^{\prime} \mathrm{E}$ and altitude 289.5 $\mathrm{m}$ above mean sea level.

The present experiment was conducted during three consecutive rabi seasons of 2016-17. Four prominent wheat cultivars, viz., Ratan GW-273 and GW-366 were used and cultivated in a factorial Randomized Block Design (RBD) with three sowing dates 1 December $\left(D_{1}\right), 11^{\text {th }}$ December $\left(D_{2}\right)$ and $21^{\text {st }}$ December $\left(D_{3}\right)$. The GDD, PTU, HTU, Intercepted PAR and HUE were computed by using following formula:

\section{Growing degree days (GDD)}

$$
\mathrm{GDD}=[(\mathrm{Tx}+\mathrm{Tn}) / 2-\text { Base temperature }]
$$

Where,

$\mathrm{Tx}=$ Daily maximum temperature

$\mathrm{Tn}=$ Daily minimum temperature

\section{Photo thermal unit (PTU)}

PTU is calculated by multiplying GDD with maximum possible sunshine hours

$$
\mathrm{PTU}=\mathrm{GDD} \times \mathrm{N}
$$

Where,

$\mathrm{N}=$ maximum possible sunshine hour.

\section{Heliothermal unit (HTU)}

HTU is calculated by multiplying GDD with actual sunshine hours (n).

$$
\mathrm{HTU}=\mathrm{GDD} \times \mathrm{X}
$$

Where,

$\mathrm{n}=$ actual sunshine hour.

\section{Heat use efficiency (HUE)}

Heat use efficiency (HUE) for total dry matter was obtained as under

$$
\operatorname{HUE}\left(\mathrm{g} / \mathrm{m}^{2} / 0 \text { day }\right)=\frac{\operatorname{Biomass}\left(\mathrm{g} / \mathrm{m}^{2}\right)}{\operatorname{GDD}(0 \text { days })}
$$

\section{Radiation use efficiency}

RUE for total dry matter was obtained as under formula

$$
\text { RUE }=\text { yield }\left(\mathrm{kg} / \mathrm{m}^{2}\right) / \mathrm{IPAR}(\mathrm{MJ} 2)
$$

Where,

IPAR is cumulative intercepted photo synthetically active radiation.

The photo synthetic active radiation can be calculated by using the following

\section{Results and Discussion}

\section{Growing degree day (GDD)}

The growing degree days accumulated by variety under various growing environment from emergence to harvesting are shown in table 1 . It is quite clear from the table that at harvest the highest value of accumulated GDD was recorded under D1 ( $1^{\text {st }}$ December) growing environment by all three varieties tasted and third date of sowing. Rajput et al. 1987 [7] and Agrawal et al. $1999^{[1]}$ were also reported similar finding.

\section{Photo thermal unit (PTU)}

Different varieties responded differently in terms of accumulated photo thermal units (PTU) at the sowing to the harvest as data sown in table 2 . The value of photo thermal unit computed during the course of investigation indicates that it was highest under D1 ( $1{ }^{\text {st }}$ December $)$ growing environment in the all the varieties tested followed by D2 and D3 growing environment at harvest stage. Overall highest value of PTU was (22570) recorded in variety GW-273 under D1 sown crop at last stage of crop followed by GW-366 (21925) and Ratan (21917).

\section{Heliothermal unit (HTU)}

The calculated value of accumulated Helio Thermal units recorded (HTU) at various stage at crop for different varieties of wheat grown in different dates is given in the Table 3. The helio-thermal units observed highest in first date of sowing under all the varieties tested followed by D2 and D3 sowing date. In overall GW-273 recorded maximum value of HTU (16005) at harvest stage followed by GW-366 (15525) and Ratan (15501).

\section{Heat use efficiency (HUE)}

Heat Use Efficiency (HUE) for different varieties under different growing environments varied considerably (Table 4). Higher HUE was observed with wheat variety Ratan followed by GW-273. Whereas minimum HUE was observed with variety GW-366. Maximum HUE was observed under D1 ( $1^{\text {st }}$ December $)$ sowing followed by D2 $\left(11^{\text {th }}\right.$ December $)$ sowing and the minimum HUE was observed under D3 (21 $1^{\text {st }}$ December) sowing.

The mean HUE in kg ha-1 degree day-1 varied from 2.21 for normal date (1st week of December) of sowing to 1.27 for vary late sowing (1st week of January) Patra and Sahu (2007). Similar findings were also reported by Rao et al. (1999) ${ }^{[8]}$. The results revealed that under normal sowing condition the crop accumulated higher amount of heat unit than late sown condition. The HUE was also higher for earlier sowings than later sowings.

\section{Radiation use efficiency (RUE)}

Radiation Use Efficiency (RUE) of different varieties recorded under different growing environments varied considerably (Table 5). On the mean basis higher RUE value was observed under D1 ( $1^{\text {st }}$ December) sowing followed by D2 (11 ${ }^{\text {th }}$ December) D3 (21 ${ }^{\text {st }}$ December) sowing. Among the varieties GW-273 showed better in terms of RUE followed by Ratan and GW-366.

In case of GW-273 maximum RUE was observed in D1 $\left(1^{\text {st }}\right.$ December) followed by D3 (21 ${ }^{\text {st }}$ December) and D2 (11 ${ }^{\text {th }}$ December). In Ratan variety the highest RUE was observed in $11^{\text {th }}$ December followed by $1^{\text {st }}$ December and $21^{\text {st }}$ December. But in case of GW-366 highest RUE value was recorded on $11^{\text {th }}$ December followed by $21^{\text {st }}$ December and $1^{\text {st }}$ December. Lowest RUE was observed under D3 (21 ${ }^{\text {st }}$ December) sowing in the all varieties. 
Table 1: Accumulated growing degree days (GDD) at different growth stages of wheat varieties under different growing environments

\begin{tabular}{|c|c|c|c|c|c|c|c|c|c|c|c|}
\hline Sowing dates & Emergence & CRI & Tillering & $\begin{array}{c}\text { Penicle } \\
\text { initiation }\end{array}$ & Booting & $\begin{array}{c}\text { Penical } \\
\text { emergence }\end{array}$ & Flowering & Milking & Dough & Maturity & Harvest \\
\hline \multicolumn{12}{|c|}{ V1 (Ratan) } \\
\hline$D_{1}-1$ December & 103 & 318 & 502 & 721 & 811 & 886 & 1118 & 1281 & 1491 & 1627 & 1876 \\
\hline $\mathrm{D}_{2}-11$ December & 91 & 267 & 448 & 707 & 787 & 850 & 1024 & 1211 & 1483 & 1684 & 1776 \\
\hline$D_{3}-21$ December & 63 & 265 & 430 & 708 & 813 & 882 & 1089 & 1265 & 1472 & 1591 & 1773 \\
\hline \multicolumn{12}{|c|}{ V2 (GW-273) } \\
\hline$D_{1}-1$ December & 103 & 318 & 502 & 706 & 795 & 871 & 1101 & 1262 & 1492 & 1792 & 1934 \\
\hline $\mathrm{D}_{2}$ - 11 December & 78 & 281 & 460 & 693 & 770 & 850 & 1061 & 1192 & 1464 & 1672 & 1885 \\
\hline$D_{3}-21$ December & 63 & 265 & 445 & 725 & 796 & 882 & 1069 & 1225 & 1491 & 1666 & 1796 \\
\hline \multicolumn{12}{|c|}{ V3 (GW-366) } \\
\hline$D_{1}-1$ December & 103 & 305 & 488 & 661 & 721 & 841 & 1066 & 1225 & 1492 & 1710 & 1865 \\
\hline $\mathrm{D}_{2}-11$ December & 78 & 267 & 434 & 648 & 723 & 819 & 1024 & 1230 & 1464 & 1672 & 1825 \\
\hline $\mathrm{D}_{3}-21$ December & 63 & 265 & 430 & 677 & 677 & 743 & 1069 & 1265 & 1491 & 1672 & 1796 \\
\hline
\end{tabular}

Table 2: Accumulated photothermal units (PTU) at different growth stages of wheat varieties under different sowing dates

\begin{tabular}{|c|c|c|c|c|c|c|c|c|c|c|c|}
\hline Sowing dates & Emergence & CRI & Tillering & $\begin{array}{c}\text { Penicle } \\
\text { initiation }\end{array}$ & Booting & $\begin{array}{c}\text { Penical } \\
\text { emergence }\end{array}$ & Flowering & Milking & Dough & Maturity & Harvest \\
\hline \multicolumn{12}{|c|}{ V1 (Ratan) } \\
\hline$D_{1}$ - 1 December & 1119 & 3468 & 5489 & 7895 & 8929 & 9791 & 12457 & 14381 & 16667 & 19661 & 21917 \\
\hline $\mathrm{D}_{2}-11$ December & 995 & 2923 & 4912 & 7834 & 8753 & 9475 & 11697 & 13715 & 16982 & 18533 & 20543 \\
\hline$D_{3}-21$ December & 690 & 2912 & 4727 & 7931 & 9130 & 9932 & 12408 & 14523 & 17229 & 18272 & 20317 \\
\hline \multicolumn{12}{|c|}{ V2 (GW-273) } \\
\hline $\mathrm{D}_{1}-1$ December & 1119 & 3468 & 5489 & 7731 & 8746 & 9618 & 12261 & 14153 & 17229 & 20977 & 22570 \\
\hline $\mathrm{D}_{2}-11$ December & 855 & 3077 & 5046 & 7671 & 8559 & 9475 & 11921 & 13492 & 16749 & 19277 & 21540 \\
\hline $\mathrm{D}_{3}-21$ December & 690 & 2912 & 4899 & 8119 & \begin{tabular}{|l|}
8934 \\
\end{tabular} & 9932 & 12172 & 14045 & 16912 & 19002 & 20556 \\
\hline \multicolumn{12}{|c|}{ V3 (GW-366) } \\
\hline$D_{1}-1$ December & 1119 & 3327 & 5341 & 7238 & \begin{tabular}{|l|}
7895 \\
\end{tabular} & 9275 & 11864 & 13704 & 17229 & 19661 & 21925 \\
\hline $\mathrm{D}_{2}-11$ December & 855 & 2923 & 4763 & 7146 & 8008 & 9118 & 11476 & 13715 & 16912 & 19524 & 20550 \\
\hline$D_{3}-21$ December & 690 & 2912 & 4727 & 7575 & 8328 & 9537 & 12172 & 14523 & 16749 & 19277 & 20530 \\
\hline
\end{tabular}

Table 3: Accumulated heliothermal units (HTU) at different growth stages of wheat varieties under different sowing dates

\begin{tabular}{|c|c|c|c|c|c|c|c|c|c|c|c|}
\hline Sowing dates & Emergence & CRI & Tillering & PI & Booting & $\begin{array}{c}\text { Penical } \\
\text { emergence }\end{array}$ & Flowering & Milking & Dough & Maturity & Harvest \\
\hline \multicolumn{12}{|c|}{$V_{1-R a t a n}$} \\
\hline $\mathrm{D}_{1}$ - 1 Dec. & 769 & 2491 & 3804 & 5299 & 6153 & 6751 & 8560 & 10118 & 12143 & 13025 & 15501 \\
\hline $\mathrm{D}_{2}-11$ Dec. & 765 & 2155 & 3194 & 5304 & 6044 & 6647 & 8146 & 9854 & 12006 & 12964 & 14612 \\
\hline $\mathrm{D}_{3}-21$ Dec. & 501 & 1792 & 2992 & 5443 & 6084 & 6645 & 8857 & 9533 & 11941 & 13881 & 14360 \\
\hline \multicolumn{12}{|c|}{$V_{2}-G W-273$} \\
\hline$D_{1}-1$ Dec. & 769 & 2491 & 3804 & 5169 & 5936 & 6364 & 8446 & 9922 & 12143 & 14832 & 16005 \\
\hline $\mathrm{D}_{2}-11$ Dec. & 652 & 2261 & 3295 & 5160 & 5880 & 6647 & 8331 & 9668 & 11822 & 13719 & 15322 \\
\hline$D_{3}-21$ Dec & 501 & 1792 & 3118 & 5605 & 6038 & 6751 & 8651 & 9918 & 11941 & 13385 & 14600 \\
\hline \multicolumn{12}{|c|}{ V3-GW-366 } \\
\hline $\mathrm{D}_{1}$-1Dec. & 769 & 2408 & 3726 & 4824 & 5299 & 6400 & 8323 & 10206 & 12098 & 13881 & 15525 \\
\hline$D_{2}-11$ Dec. & 652 & 2261 & 3194 & 4880 & 5441 & 6349 & 7955 & 10061 & 11822 & 13719 & 14635 \\
\hline$D_{3}-21$ Dec. & 501 & 1792 & 2992 & 5145 & 5771 & 6222 & 8651 & 9535 & 11941 & 13792 & 14551 \\
\hline
\end{tabular}

Table 4: Heat use efficiency (HUE) at different growth stages of wheat varieties under different growing environments

\begin{tabular}{|c|c|c|c|c|}
\hline & \multicolumn{3}{|c|}{ Heat use efficiency (g/m $\mathbf{m}^{\mathbf{2}} \mathbf{\text { deg}}$. Day) } & \\
\hline Varieties & $\begin{array}{c}\mathbf{D}_{\mathbf{1}-1} \\
\text { December }\end{array}$ & $\begin{array}{c}\mathbf{D}_{\mathbf{2}-11} \\
\text { December }\end{array}$ & $\begin{array}{c}\mathbf{D}_{\mathbf{3}} \mathbf{2 1} \\
\text { December }\end{array}$ & Mean \\
\hline Ratan & 0.47 & 0.44 & 0.41 & 0.44 \\
\hline GW-273 & 0.43 & 0.45 & 0.39 & 0.42 \\
\hline GW-366 & 0.42 & 0.39 & 0.43 & 0.41 \\
\hline Mean & 0.44 & 0.43 & 0.41 & \\
\hline
\end{tabular}

Table 5: Radiation use efficiency (RUE) at different growth stages of wheat varieties under different growing environments

\begin{tabular}{|c|c|c|c|c|}
\hline Varieties & \multicolumn{2}{|c|}{ Radition us efficiency (g MJ-1) } & \multicolumn{2}{|c|}{} \\
\hline & $\begin{array}{c}\text { D1-1 } \\
\text { December }\end{array}$ & $\begin{array}{c}\text { D } 2-11 \\
\text { December }\end{array}$ & $\begin{array}{c}\text { D3 21 } \\
\text { December }\end{array}$ & Mean \\
\hline Ratan & 1.20 & 1.25 & 1.18 & 1.21 \\
\hline GW-273 & 1.27 & 1.17 & 1.23 & 1.22 \\
\hline GW-366 & 1.10 & 1.12 & 1.02 & 1.08 \\
\hline Mean & 1.19 & 1.18 & 1.14 & \\
\hline
\end{tabular}

\section{Conclusion}

Based on the above findings it was concluded that under 1 December as it produced higher plant height, dry matter, length of spikes, number of effective tillers, test weight, harvest index, as compared to sowing on $11^{\text {th }}$ December and $21^{\text {st }}$ December. However D1 growing environment are best for getting higher yield in all wheat varieties. With respect to the heat unites viz., growing degree days, Helio thermal unit, photo thermal unit the early sowing of wheat on $1^{\text {st }}$ December $\left(D_{1}\right)$ is also suitable for wheat cultivation in Chhattisgarh plain region and variety $\mathrm{GW}-273$ is suitable cultivar for getting higher grain yield

\section{Reference}

1. Agrawal KK, Sanker U, Upadhyay AP, Gupta VK. Accumulated heat unit requirements for different phenophases of wheat (Triticum aestivum) cultivars as influenced by sowing dates at Jabalpur. Journal of Agro meteorology 1999;1(2):173-176. 
2. Anonymous. Annual report Department of Agriculture and Cooperation Ministry of agriculture Government of India Krishi Bhawan, New Delhi 2014-15.

3. Anonymous 2010. www.agridept.cg.gov.in.

4. Breiman, Graur. Wheat evolution. Israeal journal of plant science 43:85-98. Chaudhary, J. L., Patel, S. R., Verma, P.K., Manikanandan, N., Khavse, R. Thermal and radiation effect studies of different wheat varieties in Chhattisgarh plains zone under rice-wheat cropping system. MAUSAM 1995;67(3):677-682.

5. Chaudhary JL, Patel SR, Verma PK, Manikanandan N, Khavse R. Thermal and radiation effect studies of different wheat varieties in Chhattisgarh plains zone under rice-wheat cropping system. MAUSAM 1995;67(3):677-682.

6. Draganska E, Kuchar L, Szwejkowski Z. Weather-crop model selection by Cross Validation test for winter wheat cultivated in North-Eastern Poland. Acta-Scientiarum Polonorum-Agricultura 2004;3(1):29-36.

7. Rajput RP, Deshmukh MR, Paradker VK. Accumulated heat units andphenology relationship in wheat as influenced by planting dates under late sown conditions. Journal of Agronomy Crop Science 1987;159:345-349.

8. Rao VUM, Singh Diwan, Singh Raj. Heat use efficiency of winter crops in Haryana. Journal of Agrometeorology 1999;1(2):143-148. 